EGU21-7659, updated on 11 Mar 2021

https://doi.org/10.5194/egusphere-egu21-7659

EGU General Assembly 2021

(c) Author(s) 2021. This work is distributed under

the Creative Commons Attribution 4.0 License.

\title{
Ray paths of VLF/LF transmitter radio signals in the seismic Adriatic regions
}

Mohammed Y. Boudjada ${ }^{1}$, Hans Ulrich Eichelberger ${ }^{1}$, Pier Francesco Biagi ${ }^{2}$, Konrad Schwingenschuh ${ }^{1}$, Giovanni Nico $^{3}$, Maria Solovieva ${ }^{4}$, Anita Ermini ${ }^{5}$, Iren Adelina Moldovan ${ }^{6}$, Michael $^{2}$ E. Contadakis ${ }^{7}$, Aleksandra Nina ${ }^{8}$, Konstantinos Katzis $^{9}$, Mourad Bezzeghoud ${ }^{10}$, Helmut Lammer ${ }^{1}$, Patrick H.M. Galopeau ${ }^{11}$, Bruno Besser ${ }^{1}$, and Özer Aydogar ${ }^{1}$

'Institut für Weltraumforschung, Extraterrestrial Physics, Graz, Austria (mohammed.boudjada@oeaw.ac.at)

${ }^{2}$ Department of Physics, University of Bari, Bari, Italy

${ }^{3}$ Institute for Applied Mathematics (IAC), National Research Council of Italy (CNR), Bari, Italy

${ }^{4}$ Institute of the Earth Physics, RAS, Moscow, Russia

${ }^{5}$ Department of Industrial Engineering, University of Tor Vergata, Rome, Italy

${ }^{6}$ National Institute of Earth's Physics, Seismological Department, Bucharest, Romania

${ }^{7}$ Department of Surveying \& Geodesy, University of Thessaloniki, Thessaloniki, Greece

${ }^{8}$ Institute of Physics Belgrade, University of Belgrade, Belgrade, Serbia

${ }^{9}$ Department of Computer Science and Engineering, European University Cyprus, Nicosia, Cyprus

${ }^{10}$ Geophysical Centre of Évora and Physics Department, ECT, University of Évora, Évora, Portugal

${ }^{11}$ LATMOS-CNRS, Université Versailles Saint-Quentin-en-Yvelines, Guyancourt, France

We analyze the radio wave propagations of VLF/LF transmitter signals along subionospheric paths using two different reception systems localized in the Graz seismo-electromagnetic facility $(15.43 \mathrm{E}, 47.06 \mathrm{~N})$. Those systems allow the simultaneous detection of more than fifteen transmitter signals emitting in the northern (i.e. France, Germany and United Kingdom) and southern (i.e. Italy and Turkey) parts of Europe. In this work, we investigate the transmitter radio wave propagations associated with two earthquakes (EQs) which occurred, at two occasions, in nearly the same Croatian regions (Geo. Long. $=16^{\circ} \mathrm{E}$; Geo. Lat. $=45^{\circ} \mathrm{N}$ ). The first and second EQs happened, respectively, on March 22 and December 29, 2020, with magnitudes $M_{w}$ equal to 5.4 and 6.4. The use of two complementary reception systems, i.e. INFREP (Biagi et al., Open Journal of Earthquake Research, 8, 2019) and UltraMSK (Schwingenschuh et al., Nat. Hazards Earth Syst. Sci., 11, 2011), and the proximity to the epicenters lead us to characterize the behavior of the transmitter signal amplitudes particularly above the Croatian seismic regions. We analyze the amplitude variation for a given transmitter frequency starting few weeks before the earthquakes occurrences. We discuss the observed anomalies in the transmitter signals which may be considered as precursors due to the ionospheric disturbances of the transmitter ray paths above the earthquakes preparation zones. 